\title{
Applications of Wavelet Transforms to the Analysis of Superoscillations
}

\author{
Yossef Ben Ezra, Boris I. Lembrikov, \\ Moshe Schwartz and Segev Zarkovsky \\ Additional information is available at the end of the chapter
}

http://dx.doi.org/10.5772/intechopen.76333

\begin{abstract}
The phenomenon of superoscillation is the local oscillation of a band limited function at a frequency $\omega$ higher than the band limit. Superoscillations exist during the limited time intervals, and their amplitude is small compared to the signal components with the frequencies inside the bandwidth. For this reason, the wavelet transform is a useful mathematical tool for the quantitative description of the superoscillations. Continuoustime wavelet transform (CWT) of a transient signal $f(t)$ is a function of two variables: one of them represents a time shift, and the other one is the scale or dilation variable. As a result, CWT permits the simultaneous analysis of the transient signals both in the time and frequency domain. We show that the superoscillations strongly localized in time and frequency domains can be identified by using CWT analysis. We use CWT with the Mexican hat and Morlet mother wavelets for the theoretical investigation of superoscillation spectral features and time dependence for the first time, to our best knowledge. The results clearly show that the high superoscillation frequencies, time duration, and energy contours can be found by using CWT of the superoscillating signals.
\end{abstract}

Keywords: wavelet transform, superoscillations, transient signals, low-pass filter

\section{Introduction}

Superoscillating signals are band-limited signals that oscillate in some region faster than their largest Fourier component [1]. Superoscillatory functions may have interesting applications in quantum mechanics, signal processing, and optics (see, for instance, [1] and references therein). However, the superoscillation amplitude is usually so small compared to the typical values of the amplitude in non-superoscillating regions that the practical applications of the 
superoscillating functions depend on tailoring the functions in order to reduce such an effect [1]. It has been shown that the superoscillations amplitude decreases exponentially with the length of the superoscillating stretch [2]. Nevertheless, the existence of superoscillations and the possibility of encoding arbitrary amounts of information into an arbitrary short segment of a low-bandwidth signal do not contradict the information theory [2]. Taking into account the Shannon's theorem concerning the information channel capacity, it appeared to be that the superoscillatory information can be compressed to an arbitrary extent under the condition that the signal power increases exponentially with the length of the superoscillatory part of the message [2]. Superoscillations can be designed by prescribing their amplitude and/or their derivative on a grid which is denser than the Nyquist density [3]. Four different ways to constrain the signal in order to render it superoscillatory have been described in Reference [3]: (1) amplitude constraints, without any restriction on the derivative; (2) derivative constraints, without restrictions on the amplitude; (3) the amplitude and the derivative constraints on staggered grids; and (4) the amplitude and the derivative constraints on aligned grids at one half density [3]. When a set of constraints is chosen to ensure a required superoscillation, the signal is optimized by minimizing its total energy within the subspace of all the superoscillatory functions obeying the same set of constraints [2]. Superoscillations can be constructed also by using the so-called direct approach. This approach is based on a signal that is a superposition of time shifted SINC functions which ensures its band limitation [4]. Then the coefficients of the superposition are chosen by specification of the signal values on a relatively dense set of points, which forces the required superoscillations yet leaves some degrees of freedom for optimization [4]. The propagation of the temporal optical signals with a superoscillation at an absorbing resonance of a dielectric medium has been studied theoretically [5]. The absorption acts only on the Fourier components of the band limited signals, while the superoscillation is not absorbed [5]. When the signal propagates through the medium, the superoscillation revives periodically or quasi-periodically, and a superoscillatory signal may be used in order to deliver fast oscillations to a target in a dielectric medium in the frequency bands characterized by a high absorption [5].

It should be noted that it is impossible to infer the bandwidth of a finite energy signal $f(t)$ from a sampled segment of length $T$ even for a sufficiently large $T$ because there exist signals of an arbitrary small bandwidth oscillating throughout an interval of a length $T$ with an arbitrary small period [2]. The meaning is that we can make the superoscillatory part of a signal, $T$, and the corresponding frequency inside, $\omega$, arbitrarily large. This comes at a price, that the amplitude of the signal outside the superoscillatory portion is exponentially large in the number of superoscillations present in the time interval, $T$, compared to the amplitude of superoscillation $[1,2]$. In such a case, the standard Fourier analysis is not sufficient because we cannot locate the sharp pulses in the signal spectrum caused by the sharp changes of the signal in the time domain [6]. A small perturbation of the sinusoidal function $\sin (\omega t)$ or $\cos (\omega t)$ at any point of the time axis influences every point of the frequency axis and vice-versa [6]. The Fourier transform integral can be evaluated at only one frequency at a time which is not convenient for the signal processing [6]. In particular, the so-called time-frequency analysis combining both the frequency domain and the time domain analyses is necessary for superoscillation studies $[2,6]$. The short-time Fourier transform (STFT) can be used for the time-frequency analysis because it permits to obtain the 
approximate frequency contents of the time-dependent signal in the vicinity of a desired location in the time domain [6]. However, the fixed time-frequency resolution is a disadvantage of STFT because STFT can resolve properly either the low-frequency of the signal, or the high-frequency part of it [6]. The linear transform providing an efficient time-frequency resolution in any location of the time-frequency plane is the continuous-time wavelet transform (CWT) [6, 7]. CWT is defined by two real positive parameters: the scale or dilation variable $a$ and the time shift or translation $b[6,7]$. By changing the parameters $a, b$, CWT can be calculated on the entire time-frequency plane [6].

In this chapter, we constructed a family of complex valued superoscillating functions and investigated their behavior for different values of the maximum frequency $\omega_{0}$ and amplitude by using CWT with the Mexican hat and Morlet mother wavelets. We have shown that the high superoscillation frequencies, short-time durations, and energy contours can be evaluated by using CWT.

The chapter is organized as follows. The superoscillating function properties are discussed in Section 2. Some possible applications of superoscillations are reviewed in Section 3. CWT and discrete wavelet transform (DWT) definition and fundamental features are presented in Section 4. The applications of wavelet transform for the optical signal processing are briefly discussed in Section 5. The simulation results are presented and discussed in Section 6. Conclusions are presented in Section 7.

\section{The properties of the superoscillating functions}

The frequency limited functions and superoscillations occur in a number of scientific and technological applications such as foundations of quantum mechanics, information theory, optics, and signal processing which led also to work on the optimization and stability of superoscillations [8-14]. Some examples of superoscillatory functions have been proposed and investigated in the past $[1-4,8,10,11]$. In this section, we consider the generic example of the Aharonov, Popescu, Rohlich functions $f(x)$ given by [11]:

$$
f(x)=(\cos x+i g \sin x)^{n} ; g>1 ; \mathrm{n} \gg 1
$$

Here, for general, $g f(x)$ is a periodic function with a period $2 \pi$. It is easy to see from Eq. (1) that for $g=1$ we obtain from Eq. (1):

$$
f(x)=\exp (\operatorname{in} x)
$$

For small $x \rightarrow 0$, Eq. (1) yields:

$$
f(x) \approx(\exp (\ln (1+i g x)))^{n} \approx \exp (i g n x)
$$

Obviously, in the limiting case, the function determined by Eq. (3) is varying faster than the function determined by Eq. (2). Consider now the Fourier series for $f(x)$ given by [11]: 


$$
f(x)=\sum_{m=0}^{n} c_{m} \exp \left(i n k_{m} x\right)
$$

where

$$
k_{m}=1-\frac{2 m}{n} ; c_{m}=\frac{n !}{2^{n}}(-1)^{m} \frac{\left(g^{2}-1\right)^{n / 2}[(g-1) /(g+1)]^{n k_{m} / 2}}{\left[n\left(1+k_{m}\right) / 2\right] !\left[n\left(1-k_{m}\right) / 2\right] !}
$$

Equations (4) and (5) contain only wavenumbers $\left|k_{m}\right| \leq 1$ [11]. Comparison of Eqs. (3-5) shows that the function $f(x)$ demonstrates superoscillatory behavior with the degree of superoscillation defined by $g$ [11]. The function $f(x)$ Eq. (1) can be represented in an integral form [11]:

$$
f(x)=\left(\frac{g}{k(x)}\right)^{n / 2} \exp \left\{i n \int_{0}^{x} d x^{\prime} k\left(x^{\prime}\right)\right\}
$$

Here, the local wavenumber $k(x)$ is introduced given by [11]:

$$
k(x) \equiv \frac{1}{n} \operatorname{Im}\left(\frac{\partial \ln f(x)}{\partial x}\right)=\frac{g}{\cos ^{2} x+g^{2} \sin ^{2} x}
$$

The relationship Eq. (6) can be proved immediately taking into account that:

$$
\int_{0}^{x} d x^{\prime} k\left(x^{\prime}\right)=\arctan (g \tan x)
$$

and using the identities $\cos x=\left(\sqrt{1+\tan ^{2} x}\right)^{-1}$ and $\sin x=\tan x /\left(\sqrt{1+\tan ^{2} x}\right)$. The wavenumber $k(x)$ determined by Eq. (7) is varying from the superoscillatory value $k(0)=g$ to the minimum value $k(\pi / 2)=1 / g$. The superoscillatory region where $|k|>1$ is determined by the following condition [11]:

$$
|x|<x_{s}=\operatorname{arccot}(\sqrt{g})
$$

The number of oscillations $n_{o s c}$ in the superoscillatory region is given by:

$$
n_{\text {osc }}=\frac{n}{2 \pi} \int_{-\operatorname{arccot}(\sqrt{a})}^{\operatorname{arccot}(\sqrt{a})} d x k(x)=\frac{n}{\pi} \arctan (\sqrt{g})
$$

Equation (6) shows in particular that in the superoscillatory region $|k|>1$, the magnitude $|f(x)|$ is exponentially smaller than in the normal region $|k|<1$ [11]. Consequently, $n$ is the asymptotic parameter describing the number of oscillations in the superoscillatory region and the 
corresponding exponential smallness of $|f(x)|[11]$. More accurate approximation gives the following expressions for the region of fast superoscillations $x_{f_{s}}$ and the number of fast superoscillations $n_{f s}[11]$ :

$$
|x|<x_{s}=\frac{1}{\sqrt{n\left(g^{2}-1\right)}} ; n_{f s}=\frac{g \sqrt{n}}{\pi \sqrt{g^{2}-1}}
$$

Superoscillations is a week phenomenon such that there is no slightest indication of superoscillations in the power spectrum $P(k)$ of $f(x)$ [11]. Indeed, using the Fourier components Eq. (5) we obtain [11]:

$$
P(k)=\frac{n c_{m}^{2}(m=n(1-k) / 2)}{2 \sum_{0}^{n} c_{m}^{2}} \approx \frac{1}{\sigma \sqrt{2 \pi}} \exp \left\{-\frac{(k-\langle k\rangle)^{2}}{2 \sigma^{2}}\right\}
$$

where

$$
\langle k\rangle=\frac{1}{g} ; \sigma \equiv \sqrt{(k-\langle k\rangle)^{2}}=\sqrt{\frac{g^{2}-1}{2 n g^{2}}}
$$

The asymptotic spectrum Eq. (12) is a narrow Gaussian with the center at $k=g^{-1}$ representing the slow oscillations near $|x|=\pi$ [11]. The superoscillations gradually disappear getting slower and reducing to the region $|k| \leq 1$ according to the Fourier series Eqs. (4) and (5) [11].

The function $f(x)$ defined by Eq. (1) is periodic. Consequently, it may represent a diffraction grating with spatial period $\pi d$ that transforms an incident light plane wave into a propagating series of diffracted beams [11]. Such a grating transforms the wave Eq. (1) into the superoscillatory function $\Psi(x, 0)=f(x / d)$ under the following condition for the wavenumber $K: n / d<K<g n / d$ [11]. The grating produces a novel kind of super-resolution, that is, the subwavelength structure in the field with only propagating waves and without evanescent waves [11].

In the framework of the precise classical wave model, it has been shown how superoscillations can emerge and propagate into the far-field region [14]. The band-limited superoscillatory wave (the "red light") is propagating along the $x$ axis of a unidimensional (1D) waveguide with the a segment of the $x$ axis (the "window") which is opened and closed as the superoscillation passes by releasing the light pulse into the two-dimensional (2D) space corresponding to $x, z>0$ [14]. The wave traveling in the positive $x$ direction with a speed $c=1$ can be described without loss of generality by the band limited function Eq. (1) with the replacement of the argument $x$ by $(x-t) / n$ in expressions (1) and (4) [14]. This new function $f_{\text {red }}(x, t)$ is superoscillatory near $x=t$ [14]. It represents a rigidly moving polychromatic packet with associated frequencies $\omega_{m}=k_{m}$ [14]. The function $f_{\text {red }}(x, t)$ can be approximated by the following expression: $f_{\text {red.app }}(x, t)=\exp [i g(x-t)] \exp \left(\frac{(x-t)^{2}}{2 X^{2}}\right) ; X=\sqrt{\frac{n}{g^{2}-1}}$ [14]. The function $f_{\text {red.app }}(x, t)$ represents superoscillations over the interval $-2 X<x<2 X$ for $t=0$ [14]. In order 
to capture the superoscillations, the region near $x=0$ is selected with a Gaussian window of width $L$ which is opened and closed with a Gaussian switching function over an interval near $t=0$ [14]. The window must faithfully transmit the red light including the superoscillaitons [14].

For the sake of definiteness, we consider the time-dependent superoscillating signal of the type Eq. (1) assuming that:

$$
x=\frac{\omega_{0} t}{n} ; g=\frac{\omega}{\omega_{0}}
$$

Substituting relationships Eq. (14) into Eq. (1) we obtain:

$$
f_{n}\left(\omega_{0} t, \omega / \omega_{0}\right)=\left\{\cos \left(\frac{\omega_{0} t}{n}\right)+i \frac{\omega}{\omega_{0}} \sin \left(\frac{\omega_{0} t}{n}\right)\right\}^{n}
$$

Expression (15) is the signal band limited by the frequency $\omega_{0}$ with the superoscillation manifested by a single peak of a width $\Delta t=\omega^{-1}$ while the ratio $\omega / \omega_{0}$ may be arbitrary larger than unity. It should be noted that:

$$
\lim _{n \rightarrow \infty} \operatorname{Re}_{n}\left(\omega_{0} t, \omega / \omega_{0}\right)=\cos (\omega t)
$$

For finite $n$ and under the first condition of Eq. (11) which now takes the form $\omega_{0} t<$ $\sqrt{n /\left[\left(\omega / \omega_{0}\right)^{2}-1\right]}$, expression (16) reduces to the following approximation:

$$
f_{n}\left(\omega_{0} t, \omega / \omega_{0}\right) \approx \cos (\omega t)
$$

Equation (17) shows that the band limited signal Eq. (15) oscillates with a frequency $\omega$ higher than the band limit $\omega_{0}$ for the arbitrary long time depending on $n$.

\section{The possible applications of superoscillations}

Optical superoscillations can be used in the subwavelength imaging [15]. This super-resolution technology is based on a superoscillatory lens (SOL) which represents a nanostructured mask [15]. SOL illuminated with a coherent light source creates a focus at a distance which is larger than the near-field of the mask [15]. Indeed, the ability to focus beyond the diffraction limit is related to the superoscillation, since the band-limited functions in such a case oscillate faster than their highest Fourier components [11]. Superoscillatory binary masks do not use evanescent waves and focus at distances tens of wavelengths away from the mask [15]. The superoscillation-based imaging has the following advantages with respect to other technologies: (1) it is non-invasive which allows to place the object at a substantial distance from SOL; (2) it can operate at the wide range of wavelengths from X-rays to microwaves; and (3) the resolution of the SOL can be improved by refining the design, increasing the size of the 
superoscillatory mask and by increasing the dynamic range of the light detection [15]. SOL can be also used for the creation of sub-diffraction-limit optical needles [16]. An optical needle could be created by converting the central region of the SOL into an opaque area forming a shadow, and changing the diameter of the blocking region without varying the rest of SOL [16]. The possible applications of the sub-diffraction-limit optical needles are the far-field super-resolution microscopy and nanofabrication [16].

The possible applications of superoscillations for data compressions have been discussed [8]. However, the superoscillations are unstable in a way that tiny perturbations of a band-limited superoscillating function can induce very high-frequency components [8]. For this reason, the practical use of the superoscillations in imperfect communication channels is difficult [8].

\section{The fundamental properties of CWT and DWT}

There exist different types of a wavelet transform: CWT, discrete wavelet transform (DWT) [6, $7,17]$, multi-wavelets [17, 18], and complex wavelets [19]. We applied these types of wavelets to the problems related to the signal processing in optical communication systems [20-23]. We have found that CWT is the most appropriate for the analysis of superoscillations.

In this section, we consider some fundamental features of CWT. Unlike the Fourier transform and STFT, the CWT is characterized by the time and frequency selectivity [6, 7]. It can localize events both in time and in frequency in the entire time-frequency plane [6,7]. That is why CWT is unique mathematical tool for the investigation of the superoscillations where the timefrequency analysis in different regions of the spectrum is necessary as it is mentioned earlier $[2,6]$ :

The CWT $W(a, b)$ of any square integrable function $f(t)$ with respect to a wavelet $\psi(t)$ is defined as follows [7]:

$$
W(a, b) \equiv \int_{-\infty}^{\infty} f(t) \frac{1}{\sqrt{|a|}} \psi^{*}\left(\frac{t-b}{a}\right) d t
$$

Here $a, b$ are real, the asterisk denotes complex conjugation, the energy signals $f(t), \psi(t) \subset L^{2}(R)$, $L^{2}(R)$ is the set of square integrable functions such that $\int_{-\infty}^{\infty}|\psi(t)|^{2} d t<\infty$. The real- or complexvalue continuous-time function $\psi(t)$ is called the mother wavelet. It satisfies the following condition that $\int_{-\infty}^{\infty} \psi(t) d t=0[7]$.

CWT $W(a, b)$ is a function of two variables: (1) the scale or dilation variable $a$ determines the amount of time scaling or dilation and (2) the translation or time shift variable $b$ represents the shift of $\psi_{a, 0}(t)$ by an amount $b$ along the time axis and indicates the location of the wavelet window along it $[6,7]$. The inverse scaling parameter $1 / a$ is a measure of frequency [6]. 
Defining

$$
\psi_{a, b}(t)=\frac{1}{\sqrt{a}} \psi\left(\frac{t-b}{a}\right)
$$

and substituting expression (19) into Eq. (18) we obtain [7]:

$$
W(a, b) \equiv \int_{-\infty}^{\infty} f(t) \psi_{a, b}^{*}(t) d t
$$

The energy conservation law for the mother wavelet has the form for all values of $a, b$ [7].

$$
\int_{-\infty}^{\infty}\left|\psi_{a, b}(t)\right|^{2} d t=\int_{-\infty}^{\infty}|\psi(t)|^{2} d t
$$

Consider some typical mother wavelets [6, 7]. The Haar wavelet is a piecewise continuous function. It has the form:

$$
\psi(t)=\left\{\begin{array}{l}
1,0 \leq t<1 / 2 \\
-1,1 / 2 \leq t<1 \\
0, \text { otherwise }
\end{array}\right.
$$

The Mexican hat wavelet is obtained by taking the second derivative of the negative Gaussian function $-\exp \left(-t^{2}\right) / 2$ [7]. It is given by [7]:

$$
\psi(t)=\left(1-2 t^{2}\right) \exp \left(-t^{2}\right)
$$

The time dependence of the Mexican hat wavelet is shown in Figure 1.

The Morlet wavelet represents a sinusoidal function modulated by a Gaussian function given by [7]:

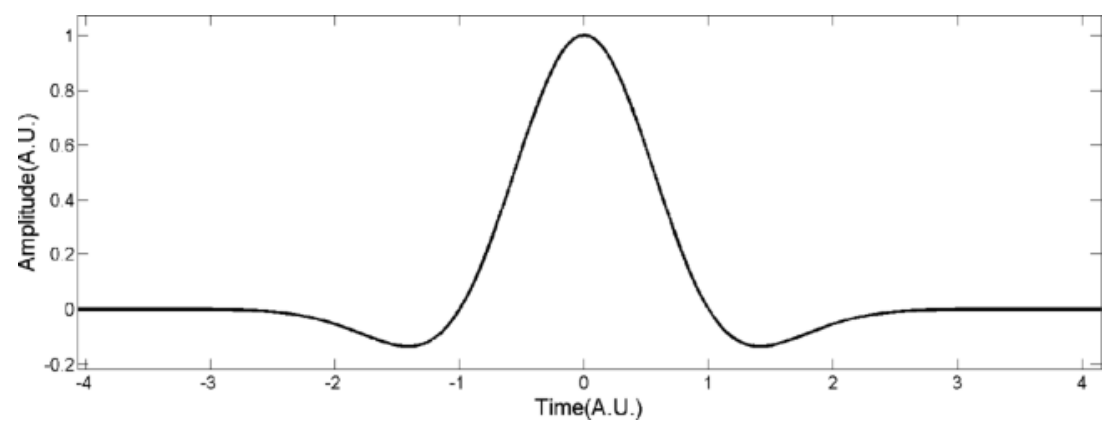

Figure 1. The Mexican hat wavelet. 


$$
\psi(t)=\exp \left(-t^{2}\right) \cos \left(\pi t \sqrt{\frac{2}{\ln 2}}\right)
$$

The time dependence of the Morlet wavelet is shown in Figure 2. It is a wavelet of an infinite duration, but most of the energy in this wavelet is confined to a finite interval [7].

CWT can be used in pattern detection and classification [6, 7]. Indeed, taking into account the definition of the inner product $\langle x(t), y(t)\rangle$ of two finite energy signals as:

$$
\langle x(t), y(t)\rangle=\int_{-\infty}^{\infty} x(t) y^{*}(t) d t
$$

one can say that CWT is a collection of the inner products of a signal $f(t)$ and the translated and dilated mother wavelets $\psi_{a, b}(t)$ for all $a, b: W(a, b)=\left\langle f(t), \psi_{a, b}(t)\right\rangle$ [7]. CWT can be also considered as the cross-correlation at lag $b$ between $f(t)$ and the mother wavelet dilated to scale factor $a$ [7]. Comparing the definition of the cross-correlation function:

$$
R_{x, y}(\tau) \equiv\langle x(t), y(t-\tau)\rangle=\int x(t) y^{*}(t-\tau) d t
$$

and CWT expression (20) we can write [7]:

$$
W(a, b)=\left\langle f(t), \psi_{a, 0}(t-b)\right\rangle=R_{f, \psi_{a, 0}}(b)
$$

The CWT is characterized by the time selectivity or the so-called windowing effect because the segment of $f(t)$ influencing the value of $W(a, b)$ for any $(a, b)$ coincides with the interval over which $\psi_{a, b}(t)$ has the bulk of its energy [7]. The CWT frequency selectivity can be described by its representation as a collection of linear, time-invariant filters with impulse responses which are dilations of the mother wavelet reflected about the time axis [7]. Indeed, using the definition of the convolution of the input signal $x(t)$ and the system impulse response $h(t)$ :

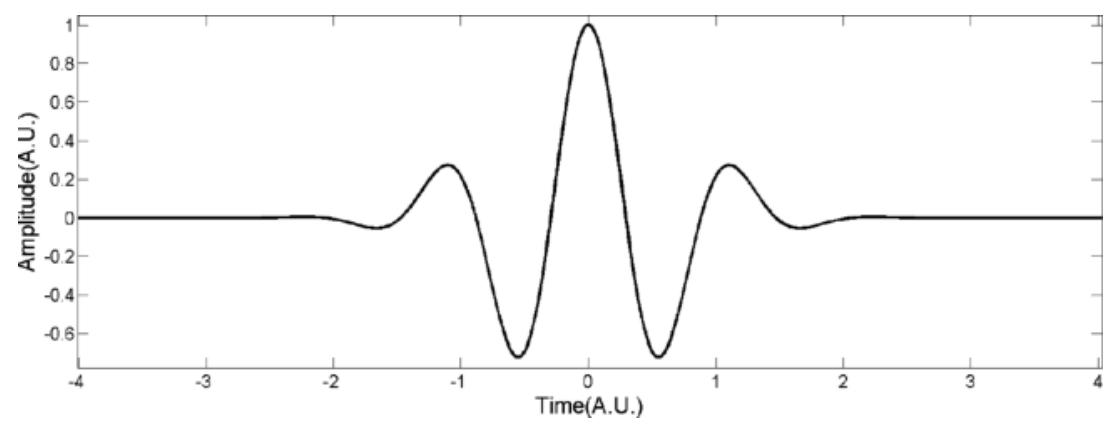

Figure 2. The Morlet wavelet. 


$$
h(t) * x(t) \equiv \int_{-\infty}^{\infty} h(\tau) x(t-\tau) d \tau
$$

we can write for the CWT:

$$
W(a, b)=f(b) * \psi_{a, 0}^{*}(-b)
$$

Consequently, for any given $a$, CWT $W(a, b)$ is the output of a filter with the impulse response $\psi_{a, 0}^{*}(-b)$ and input $f(b)$ [7]. There exists a continuum of filters characterized by the scale factor $a$ as a parameter [7]. We define the Fourier transform $\Psi(\omega)$ of the mother wavelet $\psi(t)$ :

$$
\Psi(\omega)=F\{\psi(t)\} \equiv \int_{-\infty}^{\infty} \psi(t) \exp (-i \omega t) d t
$$

Then, the corresponding $Q$ factor is determined as $Q=\omega_{c} / \Delta \omega$, where $\omega_{c}$ is center frequency of the Fourier transform Eq. (30) and $\Delta \omega$ is the 3-dB bandwidth defined as the difference between the two frequencies on either side of the peak at which $|\Psi(\omega)|^{2}$ is exactly half its peak value $|\Psi(\omega)|_{\max }^{2}[7]$. The $Q$ factor is invariant with respect to the wavelet dilation, since $F\{\psi(t / a)\}=$ $|a| \Psi(a \omega)$. The center frequency $\omega_{c}$ of $|\Psi(\omega)|^{2}$ for any $a$ is at $1 /|a|$ times the center frequency of the mother wavelet $\psi(t)$, and its $3-\mathrm{dB}$ bandwidth is $1 /|a|$ times the $3-\mathrm{dB}$ bandwidth of the mother wavelet $\psi(t)$ which gives the same value of the $Q$ factor as the one mentioned above [7]. Hence the continuum of filters mentioned above is a set of constant $Q$ bandpass filters which results in the frequency selectivity of the CWT [7]. For large values of $a$, the corresponding filter has a frequency response with a low center frequency $\omega_{0}$, and the corresponding CWT $W(a, b)$ captures the frequency content of the signal $f(t)$ around this low frequency [7]. The bandpass filter shifts to higher frequencies region with the decrease of $a$ in such a way that the CWT $W(a, b)$ at small scales contains information about $f(t)$ at the higher end of its frequency spectrum [7]. The time and frequency resolution of the CWT $W(a, b)$ are based on the duration and bandwidth of the mother wavelet $\psi(t)$, respectively. The first moments $t_{c}$ and $\omega_{c}$ of the mother wavelet $\psi(t)$ and its Fourier transform $\Psi(\omega)$, respectively, are given by [7]:

$$
t_{c} \equiv \frac{\int_{-\infty}^{\infty} t|\psi(t)|^{2} d t}{\int_{-\infty}^{\infty}|\psi(t)|^{2} d t} ; \omega_{c}=\frac{\int_{-\infty}^{\infty} \omega|\Psi(\omega)|^{2} d \omega}{\int_{-\infty}^{\infty}|\Psi(\omega)|^{2} d \omega}
$$

Expressions (31) provide the location of the center of $\psi(t)$ and $\Psi(\omega)$ along the time and frequency axes, respectively [6,7]. A measure of the wavelet duration $\Delta t$, or the root mean square (RMS) duration, and the RMS bandwidth of the wavelet $\Delta \omega$ are given by, [6, 7], respectively: 


$$
\Delta t=\sqrt{\frac{\int_{-\infty}^{\infty}\left(t-t_{c}\right)^{2}|\psi(t)|^{2} d t}{\int_{-\infty}^{\infty}|\psi(t)|^{2} d t} ; \Delta \omega}=\sqrt{\frac{\int_{-\infty}^{\infty}\left(\omega-\omega_{c}\right)^{2}|\Psi(\omega)|^{2} d \omega}{\int_{-\infty}^{\infty}|\Psi(\omega)|^{2} d \omega}}
$$

Expressions (31) and (32) can be used only for the mother wavelet $\psi(t)$ and its Fourier transform $\Psi(\omega)$ rapidly decaying in time and frequency, respectively, since the integrals in the numerators of these expressions should have finite values [7]. For the RMS duration $\Delta t_{\psi}$ and bandwidth $\Delta \omega_{\psi}$ of the mother wavelet $\psi(t)$, the RMS duration $\Delta t_{\psi}(a)$ and bandwidth $\Delta \omega_{\psi}(a)$ of its dilation $\psi_{a, 0}(t)$ are given by [7]:

$$
\Delta t_{\psi}(a) \equiv|a| \Delta t_{\psi} ; \Delta \omega_{\psi}(a)=\Delta \omega_{\psi} /|a|
$$

Combining expressions (33) we obtain:

$$
\Delta t_{\psi}(a) \Delta \omega_{\psi}(a)=\Delta t_{\psi} \Delta \omega_{\psi}=c_{\psi}=\text { const }
$$

It has been shown that the smallest time-bandwidth product is equal to $1 / 2$., and condition Eq. (34) takes the form [6, 7]:

$$
\Delta t_{\psi}(a) \Delta \omega_{\psi}(a) \geq \frac{1}{2}
$$

Equation (34) shows that the product of the wavelet duration and bandwidth is invariant to dilation. For small values of $a$, the CWT is characterized by good time resolution and poor frequency resolution because the RMS duration of the dilated wavelet is small while the RMS bandwidth of the dilated wavelet is large [7]. For large values of $a$, the time resolution of the CWT is poor, and its frequency resolution is good. The CWT provides better frequency resolution for the low-frequency region of the spectrum and poorer frequency resolution for the high-frequency region of the spectrum [7]. It can be shown that the translation parameter $b$ does not influence the mother wavelet duration and bandwidth [7].

The inverse CWT can be evaluated under the following sufficient condition for the mother wavelet Fourier transform $\Psi(\omega)[7]$ :

$$
\int_{-\infty}^{\infty} \frac{|\Psi(\omega)|^{2}}{|\omega|} d \omega \equiv C ; 0<C<\infty
$$

Then the inverse CWT has the form [7]:

$$
f(t)=\frac{1}{C} \int_{a=-\infty}^{\infty} \int_{b=-\infty}^{\infty} \frac{1}{|a|^{2}} W(a, b) \psi_{a, b}(t) d a d b
$$


The variable time-frequency resolution is an important property of the CWT which permits to use CWT for the analysis of the signals consisting of the slowly varying low-frequency components and the rapidly varying high-frequency components [7]. For this reason, the CWT is a unique tool for the study of the superoscillating signals described in Section 2.

Suppose that the dilation parameter $a$ and the translation parameter $b$ are discrete and take a form $a=2^{k}, b=2^{k} l$, where $k$ and $l$ are integers $[6,7,17]$. Then, Eq. (37) takes the form [7]:

$$
f(t)=\sum_{k=-\infty}^{k=\infty} \sum_{l=-\infty}^{l=\infty} d(k, l) 2^{-k / 2} \psi\left(2^{-k} t-l\right)
$$

The two-dimensional sequence $d(k, l)$ is defined as DWT of $f(t)$ [7]. The values of DWT $d(k, l)$ are related to the values of CWT $W(a, b)$ Eq. (18) at $a=2^{k}, b=2^{k} l$ [7]. Then DWT $W_{k l}(a, b)$ takes the form $[6,7]$ :

$$
W_{k l}(a, b)=\int_{-\infty}^{\infty} f(t) \frac{1}{2^{k / 2}} \psi^{*}\left(2^{-k} t-l\right) d t
$$

Comparison of CWT and DWT shows that the signal $f(t)$ in the both cases is expressed in terms of dilations and translations of a single mother wavelet [6]. DWT is used in the multiresolution analysis (MRA) which is based on a hierarchy of approximations to functions in $N$ various subspaces $W_{N-1}, W_{N-2}, \ldots, W_{1}$ of a linear vector space $V_{N}=W_{N-1} \oplus W_{N-2} \oplus \ldots, W_{1} \oplus V_{1}[6]$. In general case, the wavelet $\psi(t)$ providing the DWT corresponding to the MRA must satisfy the following conditions [7]:

$$
\int_{-\infty}^{\infty} \psi(t) d t=0 ; \int_{-\infty}^{\infty}|\psi(t)|^{2} d t=1 ;\langle\psi(t), \psi(t-n)\rangle=\delta(n) ;\langle\psi(t), \phi(t-n)\rangle=0
$$

Here $\delta(n)=1, n=0$ and $\delta(n)=0, n \neq 0$ and $\phi(t)$ is the scaling function $\phi(t)$ satisfying the following conditions [7]:

$$
\int_{-\infty}^{\infty} \phi(t) d t=1 ; \int_{-\infty}^{\infty}|\phi(t)|^{2} d t=1 ;\langle\phi(t), \phi(t-n)\rangle=\delta(n)
$$

The scaling function $\phi(t)$ and the wavelet function $\psi(t)$ are defined by the following equations, respectively [7]:

$$
\phi(t)=\sum_{n=-\infty}^{\infty} c(n) \phi(2 t-n) ; \psi(t)=\sum_{n=-\infty}^{\infty} d(n) \phi(2 t-n), n=0, \pm 1, \pm 2, \ldots
$$

where $c(n), d(n)$ are sequences of scalars. It is seen that the scaling function $\phi(t)$ is determined by its own dyadic dilation and translation. For this reason, the equation for $\phi(t)$ is called a dilation Equation $[6,7,17]$. It can be shown that the DWT is equivalent to filtering a signal by a band of filters with nonoverlapping bandwidths differing by a factor of 2 [17]. 


\section{The applications of wavelet transforms}

The different types of WT are widely used in different areas of mathematics and engineering [17]. The number of scientific books and articles concerning wavelet transforms (WT) applications is enormous and hardly observable. In this section, we briefly review some typical applications of wavelet transforms in optical communication systems and signal processing. Wavelet methods may complement the Fourier techniques due to their following specific features mentioned above [17]. Wavelets are functions of two parameters which represent the dilation and translation while the Fourier transform is characterized by the dilation only. In the case of wavelets, the width of the window through which the signal is observed is varying as a function of location. For a wavelet method, the window function in the time-frequency plane is nonuniform being a function of both time and frequency.

Wavelet transforms as a mathematical tool can be successfully used in the electromagnetic problems and signal processing applications [6, 7, 17-24]. Wavelet based signal processing represents a useful technique for the compression of certain classes of data demonstrating isolated band-limited properties [17]. Wavelets may be used as basis functions for the solution of Maxwell's equations in the integral or differential form [17]. Signal denoising process can be implemented by using wavelets with a smaller computational complexity as compared to the Fourier technique [17].

Wavelets can be successfully applied to signal and image processing including noise reduction, signal and image compression, signature identification, target detection, and interference suppression [6].

Wavelet packet transform (WPT) can be used in optical communications [20, 24]. WPTs are the generalization of wavelet transforms where the orthogonal basis functions are wavelet packets instead of ordinary wavelets [24]. Discrete WPT (DWPT) is used in the coherent optical orthogonal frequency division multiplexing (CO-OFDM) systems [24]. The detailed analysis of CO-OFDM communication systems can be found in $[20,24]$ and references therein. In a WPT-OFDM system, each channel occupies a wavelet packet, that is, a subcarrier in wavelet domain [24]. Inverse DWPT (IDWPT) is used at the transmitter which reconstructs the time domain signal from wavelet packets [24]. DWPT are used at the receiver in order to decompose the time domain signal into different wavelet packets by means of successive low-pass and high-pass filtering in the time domain [24].

We proposed a novel hierarchical architecture of the $1 \mathrm{~Tb} / \mathrm{s}$ transmission system based on DWPT-OFDM in order to reduce the computational complexity of the digital signal processing (DSP) algorithms [20]. We separated the low bit rate and high bit rate signal channels in such a way that the low bit rate signals are processed in the electrical domain, while the high bit rate signals are processed optically [20]. We have shown theoretically that the performance of the WPT based CO-OFDM can be significantly improved by increasing the spectral efficiency (SE) of the system and mitigating the channel chromatic dispersion [20].

Recently, some novel applications of different types of wavelet transforms have been reported. CWT can be applied for the improvement of the time-delay estimation (TDE) method in the different-wavelength based inteferometric vibration sensor in a fiber link [25]. 
The maximal overlap DWPT (MODWPT) has been used for the real-time estimation of root mean square (RMS) power value, active power, reactive power, apparent power, and power factor in power electronic systems [26].

The time-reversal (TR) technique is used for the detection and localization of objects in microwave imaging [27]. TR technique is based on an assumption that in a lossless medium, for every wave component propagating away from a source point along a certain path there exists a corresponding time-reversed wave propagating along the same path back to the original point of the source [27]. This assumption is caused by the time invariance of the Maxwell's Equations [27]. TR can achieve super-resolution by using the multipath propagation [27]. However, TR in real media is deteriorated due to the dispersion and losses [27]. A compensation method based on CWT has been proposed which can overcome both the dispersion and attenuation of the electromagnetic wave propagating in a dispersive and lossy medium [27]. In this method, the adjustable-length windows are used in such a way that the long-time windows and short-time windows are applied at low and high frequencies, respectively [27]. Wavelets depend on both the time and frequency which results in the signal decomposition into different time and frequency components. The dispersion and attenuation of these components can be compensated by different filters. Unlike the short-time Fourier transform (STFT) method, the proposed CWT method can be applied in real-life scenarios, and its resolution is about three times higher than in other methods [27].

Online monitoring and control of power grid require the accurate and fast estimation of harmonics [28]. The WT has been widely used in the estimation of time-varying harmonics [28]. In particular, undecimated WPT (UWPT) is one of multiresolution techniques characterized by redundancy and time invariance which can be implemented by a set of filter banks [28]. Unlike DWPT, the UWPT does not perform downsampling on wavelet coefficients at each decomposition level preserving time-invariant property which permits the accurate estimation of the timevarying harmonics in one cycle of the fundamental frequency [28]. The comparison of the simulation results obtained by using the UWPT based method and the experimental results shows that the UWPT algorithm has better estimation accuracy for different types of signals [28].

We for the first time to our best knowledge applied CWT to the theoretical investigation of superoscillations which requires the dynamic time-frequency analysis of the strongly localized signals. CWT appeared to be a powerful mathematical tool for the identification of the superoscillation characteristic features.

\section{The simulation results and discussion}

We theoretically investigated the superoscillations of the signal defined by the real part of expression (15):

$$
\operatorname{Re} f_{n}\left(\omega_{0} t, \omega / \omega_{0}\right)=\operatorname{Re}\left\{\cos \left(\frac{\omega_{0} t}{n}\right)+i \frac{\omega}{\omega_{0}} \sin \left(\frac{\omega_{0} t}{n}\right)\right\}^{n}
$$

The signal Eq. (38) is band limited by the maximum frequency $\omega_{0} / 2 \pi$ as it was mentioned above. Without the loss of generality, we have chosen the frequency $\omega_{0} / 2 \pi=100 \mathrm{KHz}, n=4$ 
and $n=5$. We used the Mexican hat mother wavelet Eq. (23) and the Morlet mother wavelet Eq. (24), since their oscillating temporal behavior is similar to the behavior of the superoscillating signal Eq. (38).

We investigated the scalogram of the energy contours for the spectral component of the signal Eq. (38) at the highest frequency $f\left(\omega_{0} t\right)=\cos \left(\omega_{0} t\right)$ and used the results for the analysis of the superoscillation features.

The behavior of the component with the frequency $\omega_{0} / 2 \pi$ in the time domain (lower box) and its scalogram (the upper box) are shown in Figure 3. The pseudo-frequency $\omega_{a} / 2 \pi$ shown in the scalogarm is defined as follows:

$$
\omega_{a} / 2 \pi=\omega_{c} / 2 \pi a
$$

where $\omega_{c}$ is the mother wavelet central frequency defined by the second expression (31) $[6,7]$. It is seen from Figure 3 that the homogeneous scalogram in the time-pseudo-frequency plane is strictly periodic. The maximum energy at the scalogram corresponds to the pseudofrequency $\omega_{a} / 2 \pi \approx 30 \mathrm{KHz}$.

The spectra, the temporal behavior, and the scalograms of the signal Eq. (38) for $n=4$ and $n=5$ are presented in Figures 4-7, respectively. The spectra shown in Figures 4 and 6 are obtained by using the Fourier transform. For this reason, the superoscillations are absent in the spectra shown in Figures 4 and 6, and the highest frequency corresponding to the maximum spectral amplitude is $\pm 100 \mathrm{KHz}$ in the both cases.

The scalograms shown in the upper box of Figures 5 and 7 are obtained by evaluating the CWT of the signal Eq. (38) with the Mexican hat mother wavelet Eq. (23).

The superoscillations with the time duration of about $\Delta T(n=4)=8 \mu$ s can be identified in the lower box of Figure 5. These superoscillations correspond to the frequency of about $\omega_{4} / 2 \pi=1 / \Delta T(n=4)=125 \mathrm{KHz}>\omega_{0} / 2 \pi=100 \mathrm{KHz}$. The energy contours of these superoscillations in the time-pseudo-frequency plane shown in the upper box of Figure 5 correspond to the pseudo-frequency of about $\omega_{a 4} / 2 \pi=\omega_{4} / 2 \pi a \approx(50-60) \mathrm{KHz}>\omega_{a} / 2 \pi \approx 30 \mathrm{KHz}$. Their
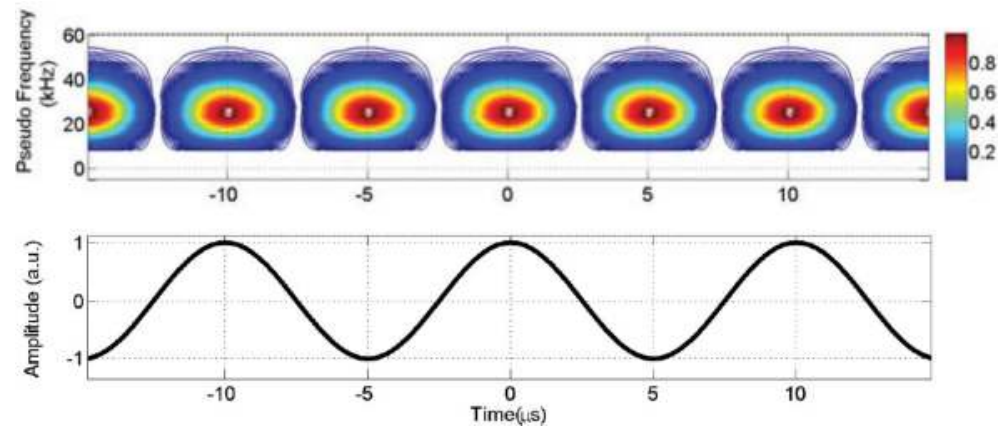

Figure 3. The time dependence (lower box) and scalogram (upper box) of the monochromatic sinusoidal signal $f\left(\omega_{0} t\right)=\cos \left(\omega_{0} t\right), \omega_{0} / 2 \pi=100 \mathrm{KHz}$. 


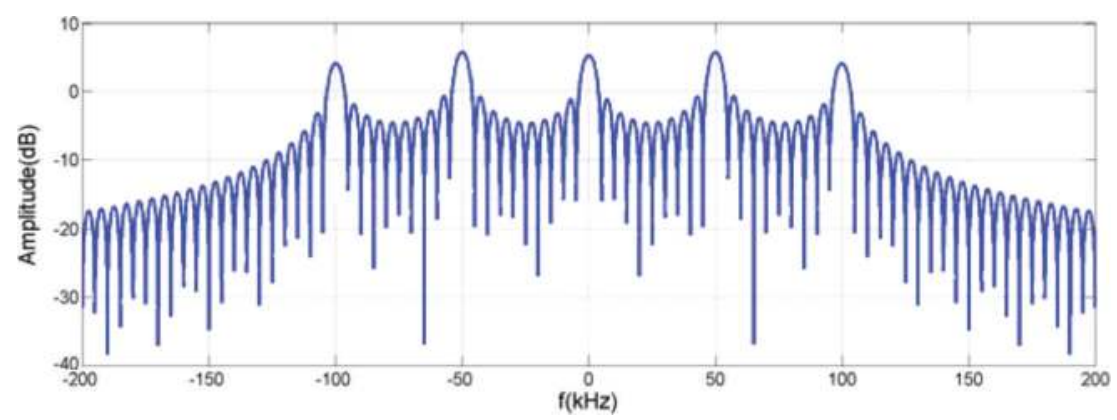

Figure 4. The spectrum of the signal $\operatorname{Re} f_{n}\left(\omega_{0} t, \omega / \omega_{0}\right)$ for $\omega_{0} / 2 \pi=100 K H z, n=4$.
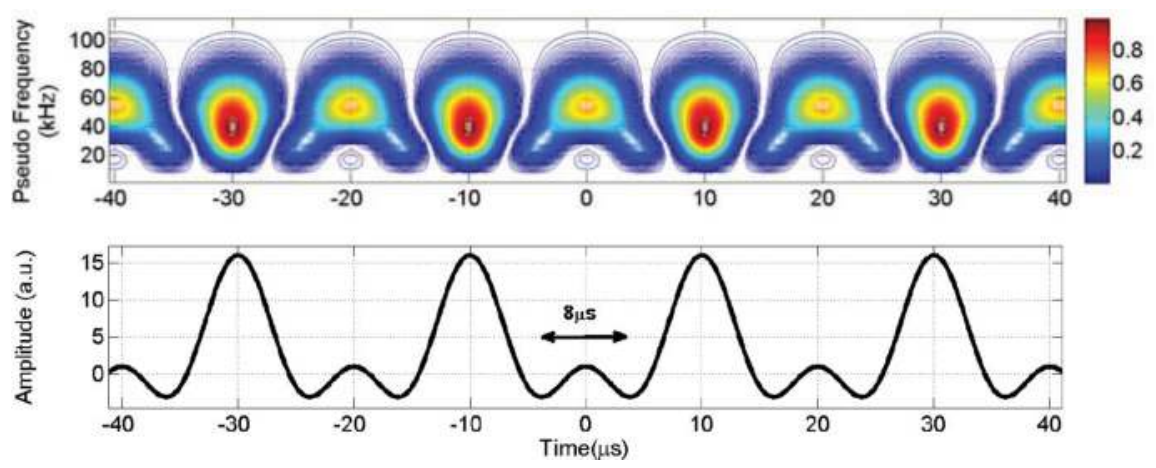

Figure 5. The time dependence (lower box) and scalogram (upper box) of the superoscillating signal $\operatorname{Re} f_{n}\left(\omega_{0} t, \omega / \omega_{0}\right)$, $\omega_{0} / 2 \pi=100 \mathrm{KHz}, n=4$, the Mexican hat mother wavelet.

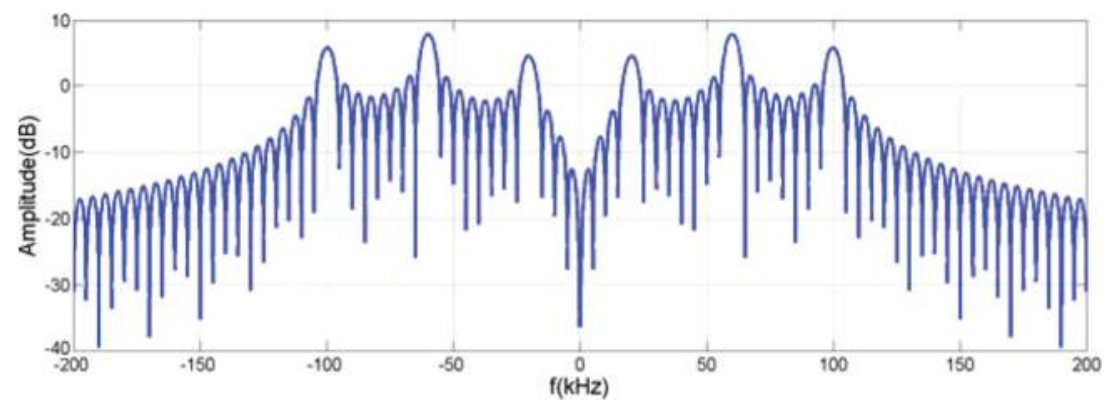

Figure 6. The spectrum of the signal $\operatorname{Re}_{n}\left(\omega_{0} t, \omega / \omega_{0}\right)$ for $\omega_{0} / 2 \pi=100 \mathrm{KHz}, n=5$.

maxima are strongly manifested at the time intervals with the center localized near $t=0, \pm 20 \mu \mathrm{s}$ and the pseudo-frequencies higher than the ones corresponding to the highest pseudo-frequency shown in the upper box of Figure 3. 

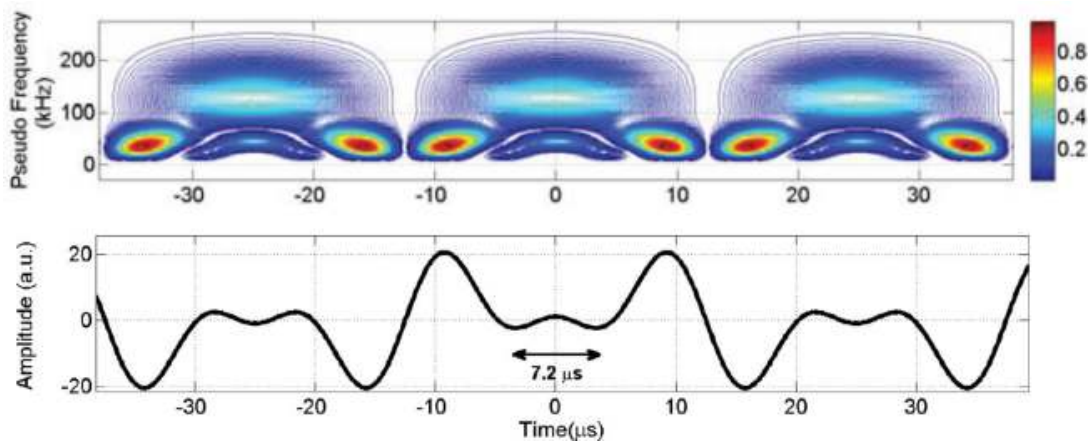

Figure 7. The time dependence (lower box) and scalogram (upper box) of the superoscillating signal $\operatorname{Re} f_{n}\left(\omega_{0} t, \omega / \omega_{0}\right)$, $\omega_{0} / 2 \pi=100 \mathrm{KHz}, n=5$, the Mexican hat mother wavelet.

It is seen from the lower box of Figure 7 that the superoscillations with the time duration of about $\Delta T(n=5) \approx 7.2 \mu s<\Delta T(n=4)$ are identified for $n=5$. They have the frequency $\omega_{5} / 2 \pi=1 / \Delta T$ $(n=5) \approx 139 \mathrm{KHz}>\omega_{4} / 2 \pi=125 \mathrm{KHz}$.

The corresponding energy contours are identified in the scalogram (upper box of Figure 7) in the time intervals localized near $t=0, \pm 25 \mu \mathrm{s}$. The maxima of the corresponding energy contours are localized at the pseudo frequency of about $\omega_{a 5} / 2 \pi=\omega_{5} / 2 \pi a \approx(100-130) \mathrm{KHz}$ $>\omega_{a} / 2 \pi \approx 30 \mathrm{KHz}$. Evidently, we can identify the higher frequency superoscillations by increasing $n$ and using CWT.

The scalograms for different mother wavelets are also different. In order to compare the CWT results consider the application of the Morlet mother wavelet Eq. (24) for the superoscillating signal $\operatorname{Re}_{n}\left(\omega_{0} t, \omega / \omega_{0}\right), \omega_{0} / 2 \pi=100 \mathrm{KHz}, n=5$.

Comparison of Figures $\mathbf{7}$ and $\mathbf{8}$ shows that the spectral features of superoscillations are pronounced at the higher pseudo-frequencies of about $(250-300) \mathrm{GHz}$, because the central frequencies of the
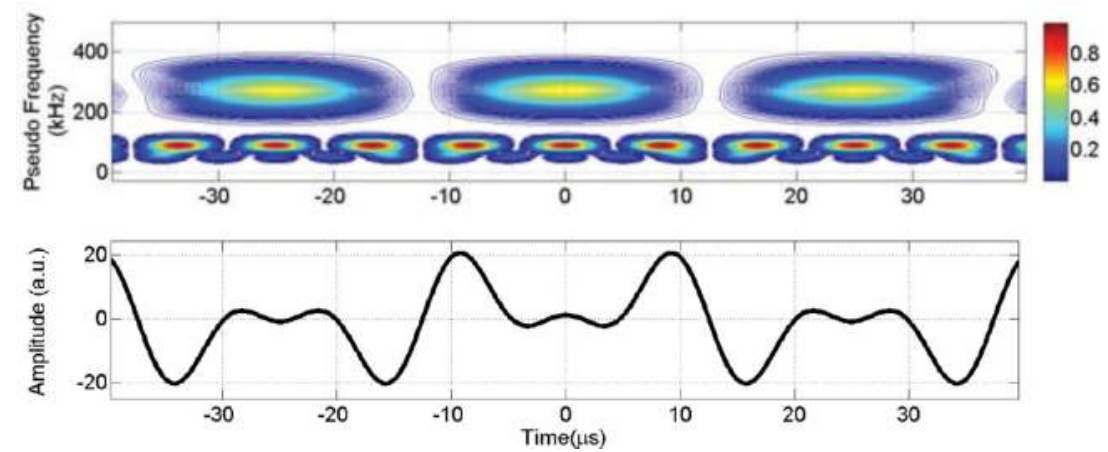

Figure 8. The time dependence (lower box) and scalogram (upper box) of the superoscillating signal $\operatorname{Re} f_{n}\left(\omega_{0} t, \omega / \omega_{0}\right)$, $\omega_{0} / 2 \pi=100 \mathrm{KHz}, n=5$, the Morlet mother wavelet. 
Mexican hat and Morlet mother wavelets are different. The real superoscillation frequencies can be obtained by pseudo-frequency multiplication by a scaling parameter $a$ according to Eq. (39).

The theoretical results of the wavelet analysis clearly show that the superoscillations with the local frequency larger that the band limit of the signal can be identified by using CWT.

\section{Conclusions}

We for the first time to our best knowledge applied CWT for the theoretical analysis of superoscillations in the time and frequency domain. We discussed the basic properties of the superoscillating signals containing the components with the frequencies larger than the maximum frequency in the signal spectrum. We also considered some possible applications of superoscillations in optics and signal processing. The superoscillating components are extremely weak and short in the time domain. They cannot be identified by the Fourier transform since they require the time-frequency analysis. We discussed the fundamental properties of CWT and DWT and their typical applications. The CWT is a unique tool for the superoscillation studies because it provides the localization of the signal both in time and in the frequency domain. We used the Mexican hat and the Morlet mother wavelets for the CWT of the sinusoidal superoscillating signal because these mother wavelets are similar to the signal oscillations. The theoretical results clearly show that the superoscillation frequency, time duration, and energy contours can be identified by using the CWT of the corresponding signal. Generally, CWT with different mother wavelets can be used for the analysis of superoscillating signals with different structures.

\section{Author details}

Yossef Ben Ezra, Boris I. Lembrikov*, Moshe Schwartz and Segev Zarkovsky

*Address all correspondence to: borisle@hit.ac.il

Department of Electrical Engineering and Electronics, Holon Institute of Technology (HIT), Holon, Israel

\section{References}

[1] Katsav E, Schwartz M. Yield-optimized superoscillations. IEEE Transactions on Signal Processing. 2013;61:3113-3118. DOI: 10.1109/TSP.2013.2258018

[2] Ferreira PJSG, Kempf A. Superoscillations: Faster than the Nyquist rate. IEEE Transactions on Signal Processing. 2006;54:3732-3740. DOI: 10.1109/TSP.2006.877642

[3] Lee DG, Ferreira PJSG. Superoscillations of prescribed amplitude and derivative. IEEE Transactions on Signal Processing. 2014;62:3371-3378. DOI: 10.1109/TSP.2014.2326625 
[4] Lee DG, Ferreira PJSG. Direct construction of superoscillations. IEEE Transactions on Signal Processing. 2014;62:3125-3134. DOI: 10.1109/TSP.2014.2321119

[5] Eliezer Y, Bahabad A. Super-transmission: The delivery of superoscillations through the absorbing resonance of a dielectric medium. Optics Express. 2014;22:31212-31226. DOI: 10.1364/OE.22.031212

[6] Goswami JC, Chan AK. Fundamentals of Wavelets. 2nd ed. New Jersey: Wiley; 2011. 359 p. ISBN: 9780470484135

[7] Rao RM, Bopardikar AS. Wavelet Transforms. 1st ed. California: Addison-Wesley; 1998. 310 p. ISBN: 0-201-63463-5

[8] Kempf A. Black holes, bandwidths and Beethoven. Journal of Mathematical Physics. 2000; 41:2360-2374. DOI: $10.1063 / 1.533244$

[9] Aharonov Y, Anandan J, Popescu S, Vaidman L. Superposition of time evolutions of a quantum system and a quantum time-translation machine. Physical Review Letters. 1990; 64:2965-2968. DOI: 10.1103/PhysRevLett.64.2965

[10] Berry MV. Evanescent and real waves in quantum billiards and Gaussian beams. Journal of Physics A: Mathematical and General. 1994;27:L391-L398. DOI: 0305-4470/94/110391

[11] Berry MV, Popescu S. Evolution of quantum superoscillations and optical superresolution without evanescent waves. Journal of Physics A: Mathematical and General. 2006;39:69656977. DOI: $10.1088 / 0305-4470 / 39 / 22 / 011$

[12] Katsav E, Perlsman E, Schwartz M. Yield statistics of interpolated superoscillations. Journal of Physics A: Mathematical and Theoretical. 2017;50:025001-1-025001-20. DOI: 10.1088/1751-8113/50/2/025001

[13] Berry MV. Suppression superoscillations by noise. Journal of Physics A: Mathematical and Theoretical. 2017;50:025003-1-025003-9. DOI: 10.1088/1751-8113/50/2/025003

[14] Berry MV, Fishman S. Escaping superoscillations. Journal of Physics A: Mathematical and Theoretical. 2018;51:025205-1-025205-14. DOI: 10.1088/1751-8121/aa9b50

[15] Rogers ETF, Lindberg J, Roy T, et al. A superoscillatory lens optical microscope for subwavelength imaging. Nature Materials. 2012;11:432-435. DOI: 10.1038/NMAT3280

[16] Diao J, Yuan W, Yu Y, Zhu Y, Wu Y. Controllable design of super-oscillatory planar lenses for sub-diffraction-limit optical needles. 2016;24:1924-1933. DOI: 10.1364/OE.24.001924

[17] Sarkar TK, Salazar-Palma M, Wicks MC. Wavelet Applications in Engineering Electromagnetics. 1st ed. Boston, USA: Artech House; 2002. 347 p. ISBN: 1-58053-267-5

[18] Cotronei M, Montefusco LB, Puccio L. Multiwavelet analysis and signal processing. IEEE Trans. Circuits and Systems-II: Analog and Digital Signal Processing. 1998;45:970-987. DOI: 1057-7130(98)04672-2

[19] Kingsbury N. Complex wavelets for shift invariant analysis and filtering of signals. Journal of Applied and Computational Analysis. 2001;10:234-253. DOI: 10.10016/acha.2000.0343 
[20] Ben Ezra Y, Lembrikov BI, Zadok A, Halifa R, Brodeski D. All-optical signal processing for high spectral efficiency (SE) optical communication. In: Das N, editor. Optical Communication. Croatia: InTech; 2012. pp. 343-366 ISBN: 978-953-51-0784-2

[21] Ben Ezra Y, Lembrikov BI. Application of complex wavelet packet transform (CWPT) in coherent optical OFDM (CO-OFDM) communication systems. In: Das N, editor. Advances in Optical Communication. Croatia: InTech; 2014. pp. 145-168 ISBN: 978-953-51-4122-8

[22] Ben-Ezra Y, Dahan D, Zarkovsky S, Lembrikov BI. High spectral efficiency (SE) OFDM system based on multi-wavelet packets. In: Proceedings of 17th International Conference on Transparent Optical Networks (ICTON 2015); July 5-9, 2015; Budapest, Hungary, (Mo. C1.3). DOI: 978-1-4673-7879-6/15

[23] Ben Ezra Y, Zadok A, Califa R, Munk D, Lembrikov BI. All-optical wavelet based orthogonal frequency division multiplexing (OFDM) system based on silicon photonic integrated components. IET Optoelectronics. 2016;10:44-50. DOI: 10.1049/iet-opt.2015.0064

[24] Li A, Shieh W, Tucker RS. Wavelet transform-based OFDM for optical communications. Journal of Lightwave Technology. 2010;28:3519-3528. DOI: 10.1109/JLT.2010.2089673

[25] Ma C, Liu T, Liu K, et al. A continuous wavelet transform based time delay estimation method for long range fiber interferometric vibration sensor. Journal of Lightwave Technology. 2016;34:3785-3780. DOI: 10.1109/JLT.2016.2583938

[26] Alves KD, Costa FB, de Araujo Ribeiro RL, de Sousa Neto CM, de Oliveira Alves Rocha T. Real-time power measurement using the maximal overlap discrete wavelet-packet transform. IEEE Transactions on Industrial Electronics. 2017;64:3177-3187. DOI: 10.1109/TIE.2016. 2637304

[27] Abduljabbar AM, Yavuz ME, Costen F, Himeno R, Yokota H. Continuous wavelet transform-based frequency dispersion compensation method for electromagnetic timereversal imaging. IEEE Transactions on Antennas and Propagation. 2017;65:1321-1329. DOI: 10.1109/TAP.2016.2647594

[28] Tiwari VK, Umarikar AC, Jain T. Fast amplitude estimation of harmonics using undecimated wavelet packet transform and its hardware implementation. IEEE Transactions on Instrumentation and Measurement. 2018;67:65-77. DOI: 10.1109/TIM.2017.2759358 